\title{
AUTOMATISIERTER UND VERNETZTER VERKEHR
}

\section{WAS KOMMT DA AUF UNS ZU?}


1.

\section{AUTOMATISIERTER UND VERNETZTER VERKEHR: WAS KOMMT DA AUF UNS ZU?}

Spätestens seit der Jahrtausendwende wird deutlich, dass Städte in Europa vor einer zunehmenden Zahl an intensiver werdenden Herausforderungen stehen. Die zwei großen globalen Treiber, die auch den gesellschaftlichen Wandel in Europa bestimmen, sind der Klimawandel sowie die globale technologische Entwicklung. Sie machen es notwendig, sich erneut grundsätzlichen Fragen zur Stadtentwicklung zu stellen. Diese beiden großen Aspekte bestimmen auch die Entwicklung der urbanen Mobilität, die vor einer grundsätzlichen Wende steht. Es werden zum einen im Zuge der Automatisierung eine Reihe von Sensoren, Fahrassistenzsystemen und Antriebssystemen entwickelt, zum anderen entstehen innerhalb der Digitalisierung - über Plattformen oder Apps gesteuert - Mobilitätsangebote (MaaS - Mobility as a Service/Mobilität als Dienstleistung), welche die Nachfrage ausdifferenzieren sowie vergrößern und dadurch neue Mobilitätsstile hervorbringen. In einer modernen mobilen Gesellschaft sind die Verkehrssysteme für die objektiv und subjektiv wahrgenommene Lebensqualität von entscheidender Bedeutung und untrennbar mit Herausforderungen der Stadtentwicklung verbunden. Neue Technologien und ein Wandel der Mobilität auf der Basis neuer Mobilitätsstile erfordern daher auch städtebauliche, stadtpolitische und stadtplanerische Neuausrichtungen von Leitbildern, Strategien, Maßnahmen, Prozessen und Instrumenten.

Neue Mobilitätstechnologien eröffnen eine ganze Palette von Möglichkeiten; die Digitalisierung kann für eine Stadtpolitik und -planung ein zusätzliches Werkzeug sein, dessen Potenziale sich erst nach und nach erschließen werden (Giffinger et al. 2018). Automatisierte und vernetzte Fahrzeuge (avF) sind ein wesentlicher Aspekt bei der Ausgestaltung von Smart City-Strategien. Zur Debatte stehen umfassende infrastrukturelle Investitionen in den Ausbau digitaler Vernetzung (5G und G5-Netze), intelligente Bordsteine oder Sensoren in der Fahrbahn oder an Ampeln (Mitteregger et al. 2019). Wie man aus der bereits länger andauernden Diskussion um das Konzept der Smart City und deren bisheriger Umsetzung ableiten kann, werden die Folgen jedoch sehr unterschiedlich eingeschätzt (Hajer 2014, Kitchin 2015, Bauriedl \& Strüver 2018, Libbe 2018). Es zeigen sich hinsichtlich der Diskurse, der Notwendigkeit für politisch-planerische Entscheidungen und der Umsetzungsprobleme gewisse Parallelitäten zu der etwas jüngeren Reflexion der avM.
Stimmt die Annahme, wie umfassend die Transformation aussehen könnte - annähernd jener durch die Einführung des Automobils - , dann wird deutlich, dass avM nicht allein eine verkehrsplanerische Aufgabe darstellt. Die Integration von avF in die Europäische Stadt (zur Verwendung des Begriffes „Europäische Stadt“ s. Kap. 3.2) sollte daher dazu genutzt werden, die Angemessenheit und den gewohnten Umgang mit bestehenden Instrumenten (Verkehrsmittel, Infrastrukturen, Plattformen und politisch-planerische Steuerungsansätze) zu reflektieren. Deshalb muss schon heute gefragt und überprüft werden, inwieweit die neuen Technologien einen Beitrag zu den aktuellen Zielen einer nachhaltigen Mobilität leisten können, ohne sich dadurch unerwünschte Nebeneffekte einzuhandeln.

\section{HOFFNUNGEN UND RISIKEN DES AUTOMATISIERTEN UND VERNETZTEN VERKEHRS}

Automatisierte und vernetzte Fahrzeuge werden in den Medien aktuell viel diskutiert. Dabei überwiegen Meldungen über technische Fortschritte sowie Anwendungsmöglichkeiten und Erwartungen, die mit diesen Mobilitätsinnovationen verbunden sind. Die Berichte werden mit futuristisch aussehenden Fahrzeugen und mit schematischen Darstellungen der Vernetzungen der Fahrzeuge in einer Smart City graphisch unterlegt. Auch in den wissenschaftlichen Veröffentlichungen dominieren Berichte über die weiterentwickelten Fahrzeugtechnologien und die neuen Potenziale aus der Vernetzung - der Fahrzeuge untereinander, mit den Smartphones und/oder dem Smart Home.

Kritische Berichte sind seltener, betreffen vor allem Fragen der Ethik, der Haftung oder der Zulassung technischer Neuerungen sowie automatisierter Fahrsysteme und bringen eine breite Skepsis der Bevölkerung zum Ausdruck. Aber auch aus der Wissenschaft kommen auf der Basis von Szenarien eine Reihe von Zweifeln ob der problemlösenden Potenziale der avM auf. Vielmehr werden Befürchtungen genannt, dass durch avF neue Probleme erzeugt bzw. bestehende verstärkt werden.

Die wissenschaftliche Auseinandersetzung um die $\mathrm{Zu}-$ kunft und die Auswirkungen des avV auf die Straßenund Siedlungsstruktur wird von den folgenden Punkten bestimmt. 
- Der Diskurs um avF ist lange von (sukzessiver oder disruptiver) technologischer Machbarkeit und/oder ökonomischer Effizienz geprägt worden (Freudendahl-Pedersen et al. 2019). Erst in jüngster Zeit wurden häufiger auch die Wechselwirkungen von ökonomischen, ökologischen und sozialen Folgen thematisiert. Dementsprechend wurde auch das Spektrum der involvierten Disziplinen erweitert, die sich dem Thema widmen (Meyer \& Beiker 2014, 2016, 2018, 2019).

- Der überwiegende Teil der Studien, der sich den Effekten des avV widmet, fokussiert auf verkehrliche Themen (z. B. steigende Effizienz oder Belastungen des Verkehrssystems, Verkehrsmittelwahl) und grenzt Fragestellungen, welche die Planung, Politik und Gesellschaft betreffen, weitgehend aus (Milakis et al. 2017, Soteropoulos et al. 2018a).

- „Selbstfahrende“ Fahrzeuge werden oftmals als „Problemlöser“ der aktuellen negativen Effekte und Nebeneffekte der (urbanen) Mobilität dargestellt: Sie sollen helfen, Staus zu vermeiden, die Unfallzahlen auf nahezu null zu senken, durch die ,intelligente“ Verkehrslenkung weniger Energie zu verbrauchen und dadurch auch die Menge der schädlichen Emissionen zu verringern. Darüber hinaus sollen bislang mobilitätseingeschränkten Personen der $\mathrm{Zu}$ gang zu eigenständiger Mobilität und damit soziale Integration (wieder) ermöglicht werden (BMVIT 2016b, 2018; Dangschat 2018; BMVI 2018; POLIS 2018).

- Während in nordamerikanischen und asiatischen (Vor-)Städten avF intensiv getestet werden und bereits erste Probebetriebe aufgenommen wurden (Lee 2018), wird in europäischen Städten erst auf ausgewählten Routen und bei geringen Geschwindigkeiten ( 8 bis $15 \mathrm{~km} / \mathrm{h}$ ) getestet (Boersma et al. 2018, Rehrl \& Zankl 2018). Bislang sind die vielseitigen, dichten und rasch wechselnden Situationen von Straßenräumen in europäischen Städten noch zu komplex, um dort avF testen zu können.

- Der gesellschaftlich-mediale Diskurs und die wissenschaftlichen Betrachtungen über den avV beziehen sich überwiegend auf die Entwicklung von Fahrzeugtechnologien und deren Konnektivität. Diese Diskussion wird von verschiedenen Fächern der Ingenieurswissenschaften und von den Herstellern von Fahrzeugen sowie von global agierenden Unternehmen der IT-Industrie und Unternehmensberatern beherrscht (Milakis et al. 2017).
- In der medialen Darstellung und im Zuge des Marketings von Smart Citys und ,smart mobility " wirken die Fahrzeuge eher futuristisch, luxuriös sowie im Stil einer Lounge. In Animationen gleiten die Fahrzeuge entweder durch eine ,gesäuberte“ Stadt, die menschenarm ist und keinerlei Hinweise auf ,urbane Herausforderungen" liefert, oder sie werden in eine weiträumige Natur hineinversetzt - Natur und Umwelt bieten dabei jedoch eine lediglich ästhetische Kulisse (Manderscheid 2018).

- In den meisten Szenarien wird fast ausschließlich auf das vollautomatisierte Fahren im SAE-Level 5 referenziert, wobei unter anderem unterschiedliche Grade der Marktdurchdringung variiert werden (meist $10 \%, 50 \%, 90 \%$; Soteropoulos et al. 2018a). Dazu werden diese Szenarien zur künftigen Entwicklung überwiegend aus einer Sichtweise auf die Interessen unterschiedlicher Akteure der Angebotsseite geprägt (Automobilindustrie, IT-Industrie, Netzwerkbetreiber; Beiker 2015).

- Daneben werden Zukunftsbilder über Straßen, Kreuzungen und Autobahnen entworfen, bei denen verschiedenen Fahrzeugen jeweils abgetrennte Spuren zugewiesen werden, was in der Realität teils zu 60 bis 80 Meter breiten innerstädtischen Straßen führen würde (NACTO 2017). Konsequenzen dieser Gestaltungen über den Verkehr hinaus, wie veränderte Aufenthaltsqualität oder steigende Trennwirkung, werden dabei weitgehend ignoriert (Mitteregger 2019, Riggs et al. 2019).

- Gerade in der Werbung, aber auch innerhalb alltäglicher Diskurse und wissenschaftlicher Publikationen steht der Pkw im Mittelpunkt künftiger Mobilitätsentwicklungen (im privaten Besitz oder im Rahmen von Sharing-Konzepten; Canzler \& Knie 2016). Vor dem Hintergrund einer allgemein zunehmenden Kritik an der Automobilität (und insbesondere der Antriebssysteme) wird durch diese Zukunftsbilder versucht, dem ,,selbstfahrenden“ Auto ein neues Image als smarter ,third space“ zu geben (neben der Wohnung und dem Arbeitsplatz).

\section{ABGRENZUNG}

Gegenstand dieser Studie sind unterschiedliche mögliche Anwendungen von avF auf Straßen im individuellen und öffentlichen Verkehr sowie in den verschiedenen Mischformen des Sharing. Die Betrachtung ist eine systematische, d. h. sie schließt alle anderen heute bekannten Modi ein. Anwendungen des Güterverkehrs und hier vor allem jene der urbanen Logistik auf der ,letzten Meile“ 
werden mitgedacht. Andere mögliche neue Transporttechnologien, von denen erwartet wird, dass sie eine Rolle im Verkehrssystem der Zukunft einnehmen könnten (Drohnen u. a.), werden in der Studie explizit ausgeschlossen.

Mit der vorliegenden Untersuchung werden die Wirkungen der avF auf die Europäische Stadt analysiert, sowohl hinsichtlich ihres normativen Rahmens, dem eine bestimmte politisch-planerische Haltung naheliegt, als auch ihres analytischen Rahmens, der Kriterien des städtischen Raums, der zukunftsfähigen Mobilität und einer heterogenen Stadtgesellschaft einschließt.

Der Betrachtungshorizont ist die zeitlich naheliegende Phase des Übergangs. Die Handlungsfelder (s. Kap. 6.3) betreffen Weichenstellungen, die in der Stadtentwicklung in den nächsten fünf bis zehn Jahren eingeleitet werden sollten. Es wird davon ausgegangen, dass avF während dieser Phase aufgrund der Heterogenität der Straßenräume und deren Nutzungen nicht gleichmäßig über die Siedlungsstruktur eingesetzt werden können. Damit rücken Phänomene eines sozial und räumlich selektiven Einsatzes des avV in den Mittelpunkt der Betrachtung. Das erfordert eine räumlich und sozial differenzierte Analyse, für die hier erste Schritte gesetzt werden. Gleichzeitig wird ein umfangreicher Forschungsbedarf ersichtlich.

Die allgemeine Debatte über avM konzentriert sich allerdings, wie bereits beschrieben, vor allem auf den technischen Fortschritt (Wann werden welche Assistenzsysteme verfügbar sein?) und auf die futuristische Gestaltung der Fahrzeuge, insbesondere der Pkws. Die Assistenzsysteme werden danach eingeordnet, wie die Mensch-Maschine-Schnittstelle der Verantwortung über das Führen des Fahrzeugs aufgeteilt ist. Im internationalen Maßstab orientiert man sich am SAE-System (SAE International, ehemalige Bezeichnung: Society of Automotive Engineers) bzw. der SAE-Norm J3016, in Deutschland am System des BASt (Bundesamt für Straßenwesen). Ein großer Teil der bisherigen Studien und vor allem der Publikationen in den Medien beschränkt sich auf diese Einstufungen und konzentriert sich hinsichtlich der Möglichkeiten auf das vollautomatisierte Fahren, dem SAE-Level 5. Im Rahmen dieses Berichts ist jedoch das hochautomatisierte Fahren Gegenstand der Betrachtung, der sogenannte Level 4 (SAE 2019, s. Kap. 4).

Auch die Frage des Zeitpunkts der Zulassung entsprechend automatisierter Fahrzeuge nimmt einen Teil der öffentlichen wie wissenschaftlichen Debatte ein. Es geht also um einen doppelten internationalen Wettbewerb: einen unter den Unternehmen der klassischen Automobil- und IT-Branche hinsichtlich der Technologie und einen zwischen den Nationalstaaten resp. nachgeordneten Gebietskörperschaften (Bundesstaaten, Bundesländer und Städte) um die Zulassungen im Straßenverkehr.

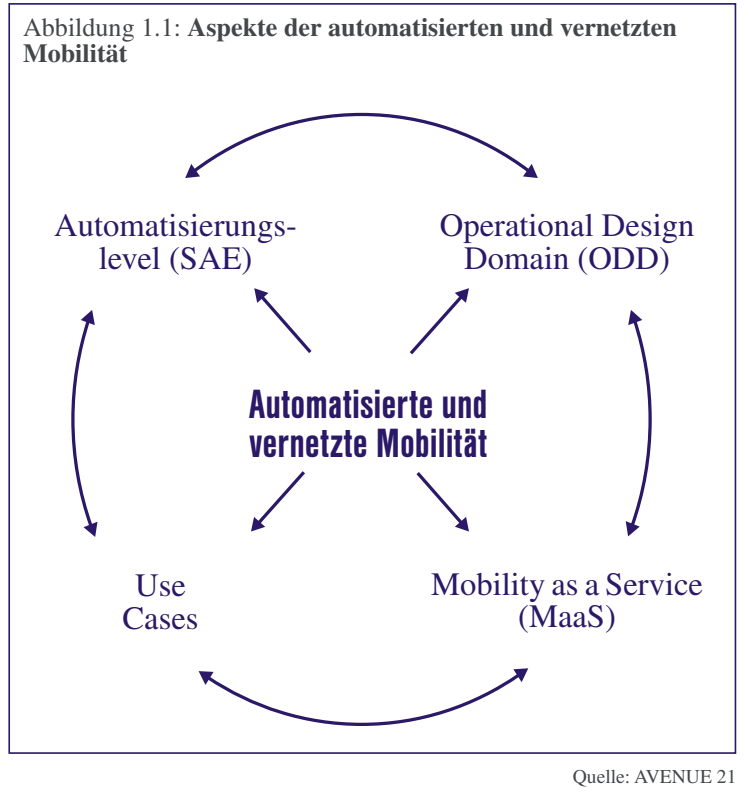

Die Zulassung unterschiedlicher Automatisierungsgrade hängt vor allem von den Einsatzgebieten (ODD - Operational Design Domain) wie Autobahnen, ländlichem Raum, innerstädtischen Quartieren oder Betriebsgeländen sowie weiteren Umweltbedingungen wie der Tageszeit oder dem Wetter ab. Aufgrund unterschiedlicher Komplexität der Verkehrssituationen, aber auch der Verfügbarkeit von leistungsfähigen Internetverbindungen werden die Zulassungen automatisierter Fahrzeuge im zeitlichen Ablauf vor allem davon abhängig sein, wie bestimmte Verkehrssituationen im gemischten Verkehr sicher organisiert werden können.

Aufgrund des Klimawandels und der überfüllten Straßenräume geht es in europäischen Stadtregionen aber gleichzeitig auch darum, eine Verkehrswende einzuleiten - dazu sind neben neuen postfossilen Antriebsformen neue Mobilitätskonzepte und Verhaltensänderungen notwendig. Digitale Plattformen werden zunehmend eingerichtet, um verschiedene Fahrzeuge für unterschiedliche Wege zu buchen, zu nutzen sowie um Kosten berechnen und eine gewisse Kontrolle ausüben zu können - MaaS. Insbesondere dem Car- und Ride-Sharing wird hierbei eine prägende Rolle zugeschrieben. Ziel der Verkehrswende ist also nicht, ein aktuelles Fahrzeug gegen ein ,,intelligenteres“ auszutauschen, sondern vor allem die urbane Mobilität völlig neu aufzusetzen, indem (1) Verkehr vermieden, (2) der Verkehr auf den Umweltverbund verlagert (aktive Mobilität - zu Fuß gehen und Rad fahren - und öffentlicher Personenverkehr) und (3) der öffentliche Raum in seiner Funktion als Aufenthaltsort verbessert wird (3-V-Strategie). Ein solcher Wandel hätte auch bemerkenswerte Auswirkungen auf das Gesicht der Europäischen Stadt.

Da in der Regel von den genannten Aspekten (Automatisierungslevel, ODD, Use Cases und MaaS) nur Ausschnitte - und diese eher parallel und nicht aufeinander 
bezogen - diskutiert oder in Szenarien berücksichtigt werden, erscheint es notwendig, die vier angesprochenen Aspekte immer im Zusammenhang zu betrachten (s. Abb. 1.1).

Da das Projekt AVENUE21 nicht darauf ausgelegt ist, künftige Entwicklungen der Mobilität in europäischen Städten zu quantifizieren, wurde auch kein klassisches ,impact assessment“ angestrebt. Es geht demnach vielmehr darum, für einen erweiterten Kreis von AkteurInnen der Stadt- und Mobilitätsplanung Möglichkeiten der aktiven Gestaltung des bevorstehenden Wandels zu entwickeln und zu belegen.

Nicht nur wegen der weitreichenden Folgen der avM, sondern letztlich auch, weil insbesondere bei weiten Teilen der Bevölkerung, (kritischen) SozialwissenschaftlerInnen und gerade auch auf kommunaler verkehrspolitischer Ebene Skepsis gegenüber den Auswirkungen des avV herrscht, ist es geboten, sich bereits jetzt mit den Bedingungen der Implementierung von avF auseinanderzusetzen, um negative Effekte bezüglich einer nachhaltigen Stadtentwicklung resp. für bestimmte Teilräume und soziale Gruppen zu vermeiden. Daher ist es das Ziel des AVENUE21-Projekts, für die lokale/regionale politisch-planerische Verwaltung alternative Szenarien zu entwickeln, woraus nicht nur der unmittelbare Handlungsbedarf ersichtlich wird, sondern auch mögliche Auswirkungen unterschiedlicher Handlungsprämissen verdeutlicht werden.
Open Access Dieses Kapitel wird unter der Creative Commons Namensnennung 4.0 International Lizenz (http://creativecommons.org/licenses/by/4.0/deed.de) veröffentlicht, welche die Nutzung, Vervielfältigung, Bearbeitung, Verbreitung und Wiedergabe in jeglichem Medium und Format erlaubt, sofern Sie den/die ursprünglichen Autor(en) und die Quelle ordnungsgemäß nennen, einen Link zur Creative Commons Lizenz beifügen und angeben, ob Änderungen vorgenommen wurden.

Die in diesem Kapitel enthaltenen Bilder und sonstiges Drittmaterial unterliegen ebenfalls der genannten Creative Commons Lizenz, sofern sich aus der Abbildungslegende nichts anderes ergibt. Sofern das betreffende Material nicht unter der genannten Creative Commons Lizenz steht und die betreffende Handlung nicht nach gesetzlichen Vorschriften erlaubt ist, ist für die oben aufgeführten Weiterverwendungen des Materials die Einwilligung des jeweiligen Rechteinhabers einzuholen.

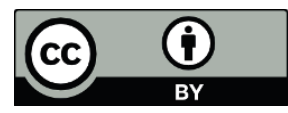

